\title{
A signature of glucose metabolism and DNA methylation improves prognostic prediction for urinary bladder cancer
}

\section{Zhuonan Liu}

Department of Urology, First Hospital of China Medical University, Shenyang, China.

\section{Tianshui Sun}

Department of Obstetrics and Gynecology, Shengjing Hospital of China Medical University, Shenyang, China.

\section{Zhe Zhang}

Department of Urology, First Hospital of China Medical University, Shenyang, China.

\section{Jianbin Bi}

Department of Urology, First Hospital of China Medical University, Shenyang, China.

\section{Chuize Kong ( $\nabla$ kongchuize_cmu@sina.cn )}

Department of Urology, First Hospital of China Medical University, Shenyang, China.

https://orcid.org/0000-0002-0875-2173

\section{Research}

Keywords: bladder cancer; glucose metabolism; DNA methylation; prognostic factor; TCGA; UCHL1; PYCR1

Posted Date: May 6th, 2020

DOl: https://doi.org/10.21203/rs.3.rs-25944/v1

License: (c) (1) This work is licensed under a Creative Commons Attribution 4.0 International License. Read Full License 


\section{Abstract}

Background: Glucose metabolism and DNA methylation have been previously proved to play important roles in cancers. However, few studies have explored the potential connections between abnormal glucose metabolism and DNA methylation in tumor progression. In this study, we aimed to screen out glucose metabolism-related genes that were DNA methylation associated in order to establish a promising signature for the prognosis of bladder cancer (BLCA).

Methods: With transcriptome data of BLCA samples from The Cancer Genome Atlas (TCGA) and methylation data downloaded from TCGA data base 450K microarray, glucose metabolism-related genes that were simultaneously methylation modulated and survival associated were obtained and a prognostic signature was established. Gene set enrichment analysis (GSEA) and weighted gene co-expression network analysis (WGCNA) were performed for identification of gene and pathway enrichment. Two most methylation-related risky genes were selected to conduct functional validations.

Results: 18 target genes were identified and the samples were survival-significantly divided into two groups by using the signature. Via multivariate Cox analysis, we considered the signature an independent prognostic factor. Several biological processes and signaling pathways were enriched in the high-risk group according to GSEA results and three modules of genes were identified by WGCNA. We demonstrated that UCHL1 and PYCR1 promoted proliferation, migration and invasion ability of bladder cancer cells.

Conclusions: The signature based on the 18 target genes could serve as an independent prognostic factor for urinary bladder cancer patients.

\section{Background}

Urinary bladder urothelial carcinoma (BLCA), one of the most common malignancies in male population, was reported to result in 81,190 new cases and 17,240 deaths estimated in the United States in 2018 [1]. It has become a disturbing issue for urologists to deal with in clinical treatment process with its high morbidity and mortality rate mainly due to its reoccurring tendency and chemoresistance [2-4]. One of the reasons of relapse tendency of bladder cancer is considered lack of predictive and prognostic biomarkers, which has been a new challenging strategy for researchers and urologists.

Dramatic changes in energy metabolism and nutrient uptake are commonly observed in various cancer cells with enhanced cell proliferation and growth to support. As one of the most important nutrients for cancer cells uptake to maintain unrestricted proliferation, not only can glucose be utilized to synthesize ATP but also to provide cancer cells with carbon source [5-6]. One of the most well-known glucose metabolic reprograming forms is "the Warburg effect" [5, 7], referring to the phenomenon that most cancer cells generate ATP by glycolysis even under aerobic conditions while it takes place only in nonaerobic conditions for normal cells. Altered expressions of many rate-limiting enzymes participate in activation of Warburg effect [5] and a large number of oncogenes and tumor-suppressors are involved in 
a variety forms of energy reprogramming processes [8-12]. However, despite the progress previous studies have achieved, we still lack comprehensive analysis and summary of glucose metabolism-related genes especially those correlated to cancer prognosis, on the basis of which potential new diagnosis and treatment targets are likely to be established.

In previous studies, abnormal DNA methylation has been proved to be related to cancers and other diseases by regulating genomic transcription. Usually detected in highly and moderate repeated DNA sequences, hypomethylation can result in chromosome instability leading to activated oncogenes [1315], while DNA hypermethylation in the promotor region can lead to silencing of some tumor suppressing genes $[13,16]$. For some genes, methylation has been found to be responsible for early carcinogenesis [17]. Thus, it has become a trend for DNA methylation to act as early diagnostic and prognostic biomarkers for tumors [18-20] because of its relative stability [21] and easy access to detection in blood. For instance, several studies have proved that hepatocellular carcinoma patients could be discriminated from healthy individuals with high sensitivities by detecting methylation of CpGs [22-24]. An FDA approved project aimed at early diagnosis of colorectal cancer by detecting promotor methylation status of SEPT9 in blood and the sensitivity and specificity were found to be 36.6 to $95.6 \%$ and 81.5 to $99.0 \%$ [25]. Considering the critical role glucose metabolism plays in cancer progressions, we intended to establish a new prognostic indicator for bladder cancer patients by exploring the associations between DNA methylations and glucose metabolism-related genes.

In our study, TCGA BLCA cohort was used as study object. We screened out 46 glucose metabolismrelated genes that were linked to overall survival, among which 18 genes' expressions were significantly correlated to their corresponding promotor methylation strengths. A signature based on the 18 genes was established to predict the BLCA prognosis. The enrichments of co-expressive genes, biological functions and signaling pathways were constructed and prove the accuracy of our bioinformatic analyses and to provide experimental bases for further research.

\section{Materials And Methods}

\section{Glucose metabolism-related gene set}

From the Molecular Signatures Database (http://www.broad.mit.edu/gsea/msigdb/), we selected all the pathways related to glucose metabolism with relevant genes included.

\section{Patient Samples}

The RNA-seq transcriptome data of Bladder Urothelial Carcinoma (BLCA) cohort was downloaded from The Cancer Genome Atlas (TCGA) data base (https://portal.gdc.cancer.gov/) along with corresponding clinical information. 


\section{Consensus Clustering Analysis}

Cancer samples from TCGA BLCA cohort included were clustered into groups by consensus expression of 30 glucose metabolism-related genes with the largest values of median absolute deviation (MAD) by using "ConsensusClusterPlus" in R program 3.3.2 (www.rproject.org). Chi-square test was used to compare the distribution of age, tumor grade, pathology stage, lymph node positivity, metastasis positivity and neoplasm grade between different clusters.

\section{Identification Of Survival-related Differentially Expressed Genes (degs)}

300 and 100 samples from TCGA BLCA cohort were randomly divided into training set and validation set, respectively. All the steps of gene screening were carried out in training set. Differentially expressed genes (DEGs) were screened out from all the glucose metabolism-related genes by comparing bladder cancer patients with overall survival time less than 1 year with those who lived longer than 3 years. $P<0.05$ was the considered the criterion. With the use of a univariate Cox proportional hazard regression analysis, we analyzed all the glucose metabolism-related genes to obtain genes remarkably correlated with patients' survival time with $p<0.05$ as the main cutoff point. The overlapping genes gained from the two steps above were considered survival-related DEGs.

\section{Analysis Of Dna Methylation Status}

Methylation data was downloaded from TCGA database 450K microarray. The correlations between the expressions of survival-related DEGs and the strengths of promoter methylation probes of corresponding genes were analyzed to divide the probes into significant probes and non-significant probes after z-score normalization with $P<0.05$ as criterion. Obtained genes were considered target genes for further steps of our study.

\section{Analysis Of Prognostic Signature}

The risk score of each patient in the training set and the validation set was calculated by using gene expression values and regression coefficients $(\beta)$ which were generated from the univariate Cox proportional hazard regression analysis, according to the formula below: risk score $=\beta$ (gene 1$) \times$ gene 1 expression $+\beta$ (gene 2$) \times$ gene 2 expression $+\beta$ (gene 3$) \times$ gene 3 expression $+\ldots+\beta($ gene $n) \times$ gene $n$ expression. Patients were divided into high-risk group and low-risk group according to the median risk score as the cutoff point. The overall survival difference between the two groups in the training set or the validation set were calculated by using Kaplan-Meier estimate. The time-dependent receiver operating characteristic (ROC) curve was used the survivalROC package (https://CRAN.Rproject.org/package=survivalROC) to assess the accuracy of prognostic signature. A multivariate Cox 


\section{Gene Set Enrichment analysis (GSEA) and weighted gene co-expression network analysis (WGCNA)}

With the use of the molecular signatures database (MSigDB) of C2 CP: Canonical pathway gene set collection, Gene Set Enrichment Analysis (GSEA) (http://software.broadinstitude. org/gsea/index.jsp) was performed in TCGA BLCA dataset. The parameter of permutations was at 1,000 and FWER adjusted $P$-value $<0.02$ was considered the significance threshold. Hub gene identification and co-expression analysis were performed by co-expression network analysis WGCNA [26], which was visualized by Cytoscape [27].

\section{Cell Lines And Cell Transfection}

Bladder cancer cell lines, UM-UC-3 and T24, were purchased from the Chinese Academy of Sciences Cell Bank (China). At $37{ }^{\circ} \mathrm{C}$ in an atmosphere of $5 \% \mathrm{CO} 2$, the two cell lines were cultured in RPMI 1640 (Hyclone; GE Healthcare) supplemented with 10\% fetal bovine serum (FBS; Biological Industries, BeitHaEmek, Israel). Small interfering RNAs (siRNAs) designed to respectively target the UCHL1 and PYCR1 were ordered from JTSBIO Co. (China) and the sequences were as follows: si-UCHL1 (sense: GGCCAAUAAUCAAGACAAATT; anti-sense: UUUGUCUUGAUUAUUGGCCTT); si-PYCR1(sense: GCCCACAAGAUAAUGGCUATT; anti-sense: UAGCCAUUAUCUUGUGGGCTT).

\section{Rna Extraction And Quantitative Real-time Pcr}

RNAiso Plus (Takara Biotechnology, Dalian, China) was used to isolate total RNA from cells. cDNA was generated by reverse transcribed total RNA with the use of Prime Script RT Master Mix (Takara Biotechnology, Dalian, China) according to the manufacture's protocol. RT-qPCR was performed with a SYBER Premix Ex Taq ${ }^{{ }^{T M}}$ Kit (Takara Biotechnology, Dalian, China) by using a Thermal Cycler Dice ${ }^{\mathrm{TM}}$ Real Time TP800 system (Takara, Kyoto, Japan) to conduct all the analyses. The relative expression level of each gene was analyzed by using the $2^{-\triangle \triangle C T}$ method normalized to GAPDH. The primer sequences for each gene were as follows: UCHL1 (forward: AATGTCGGGTAGATGACAAGGT; reverse:

GGCATTCGTCCATCAAGTTCATA), PYCR1 (forward: TGGCTGCCCACAAGATAATGG; reverse: CGTGACGGCATCAATCAGGT) and GAPDH (forward: ACAACTTTGGTATCGTGGAAGG; reverse: GCCATCACGCCACAGTTTC).

\section{Cell Proliferation Assay}


Cell proliferation ability was measured by using a BeyoClick ${ }^{\mathrm{TM}}{ }_{\text {EdU-48 }} 48$ cell proliferation kit (Beyotime Biotechnology, China). Transfected cells were co-incubated with EDU working solution (1:1000) in 6-well plates at $37^{\circ} \mathrm{C}$ in an atmosphere of $5 \% \mathrm{CO}_{2}$ for 2 hours, after which the culture medium was removed and $4 \%$ paraformaldehyde was added for fixation at room temperature for 20 minutes. Then cells were incubated at room temperature for 15 minutes with $0.3 \%$ Triton X-100 in PBS, followed by incubation with click reaction solution according to the manufacture's protocol. Images were obtained by A fluorescence microscope (Olympus Corporation, Japan).

\section{Cell Migration And Invasion Assay}

24-well polycarbonate filters with 8- $\mu$ m pores (Corning Costar, Corning, NY, USA) and were used to detect cell migration ability and invasion ability. $600 \mu \mathrm{l} 1640$ medium containing 10\% FBS was added into each bottom chamber. Certain numbers of transfected cells $\left(1.5 \times 10^{4}\right.$ cells per well in $200 \mu$ l serum-free 1640 medium for cell migration, $3 \times 10^{4}$ cells in $200 \mu \mathrm{l}$ serum-free 1640 medium per well for cell invasion) were added into the upper chambers of the filters. Polycarbonate filters were coated with $50 \%$ Matrigel (BD, San Diego, CA, USA) for cell invasiveness detection while filters without Matrigel were used to measure cell migration. After incubated at $37^{\circ} \mathrm{C}$ in an atmosphere of $5 \% \mathrm{CO}_{2}$ for 24 hours, cells remaining in the upper membranes were removed while cells that had migrated to the lower side were fixed by $4 \%$ paraformaldehyde and stained by $1.0 \%$ crystal violet. Pictures were obtained by using microscope and cell counting was conducted by ImageJ.

\section{Immunoblotting Assay}

Total protein was extracted from cells by using RIPA lysis buffer containing 1\% PMSF. Bicinchoninic acid assay (Beyotime Institute of Biotechnology) was applied to determine protein concentrations. Equal amounts of denatured protein ( $30 \mu \mathrm{g} / \mathrm{lane}$ ) were separated by $10 \%$ SDS-polyacrylamide gel electrophoresis and were transferred to onto polyvinylidene fluoride (PVDF)membranes. Blocked by $5 \%$ fat-free milk in TBST solution, membranes were incubated with anti-N-cadherin (1:5,000, ab76011, abcam, USA ), anti-beta-catenin (1:1,000, 8480S, CST, USA), anti-CyclinD1 (1:1,000, 55506S, CST, USA), anti-GAPDH $\left(1: 1,000 ; 5174 \mathrm{~S}\right.$; CST, USA) antibodies at $4{ }^{\circ} \mathrm{C}$ overnight. After being washed by TBST solution for 3 times, the membranes were incubated with secondary antibodies at $37^{\circ} \mathrm{C}$ for 1 hour and images were captured by using EasySee Western Blot kit (Beijing Transgen Biotech, Beijing, China) and a chemiluminescence system (Bio-Rad, CA, USA).

\section{Statistical analysis}

All statistical analyses for bioinformatic analysis were performed by the $\mathrm{R}$ software ( $\mathrm{R}$ version 3.3.2). As for functional validations, GraphPad Prism version 7.0 (La Jolla, CA, USA) was used for statistical 
analyses. Student's t test was performed for comparisons. Each of the experiments were conducted for at least three times and each $P$ value $<0.05$ was considered to indicate significantly statistically.

\section{Results}

\section{BLCA data acquisition}

The flowchart of the study procedure was shown in Fig. 1. Twelve pathways linked to glucose metabolism were chosen with a total of 606 glucose metabolism-related genes included for following study. The RNA-seq transcriptome data of 405 bladder cancer samples and 19 normal bladder samples from TCGA BLCA cohort was collected and corresponding DNA methylation data was obtained from TCGA 450 k microarray.

\section{Consensus clustering of glucose metabolism-related genes identified two main clusters of BLCA samples}

With the increase of $\mathrm{k}$ value, the 405 bladder cancer samples were gradually and steadily divided into two main large clusters by 30 glucose metabolism-related genes with the largest MAD values, the light green cluster and the light blue cluster (Fig. 2a-j), which suggested that glucose metabolism could play a potential role as an index in classifying bladder cancer patients. Then we tried to categorize the 30 genes into two clusters according to their expression trends, namely glucose cluster 1 and glucose cluster 2 . The result showed 405 samples were obviously divided into two clusters, cluster $A$ and cluster $B$, which were characterized with significant differences on the expressions of cluster 1 genes and cluster 2 genes (Fig. 2k). By measuring the associations between the clustering and clinicopathological features, we detected significant difference between cluster 1 and cluster 2 for neoplasm grade $(P=2.999 \mathrm{e}-06)$, while no obvious differences were found for all of the other parameters (Table. 1), which indicated that compared to current indexes, it's a relatively independent but effective method to categorize subtypes of bladder cancer samples by using glucose metabolism-related genes and it's worth further exploring. 
Table 1

Clinicopathological characteristics of bladder cancer patients by consensus clustering analysis.

\begin{tabular}{|llll|}
\hline Characteristics & Clust A $(\mathrm{N}=156)$ & Clust $\mathrm{B}(\mathrm{N}=249)$ & P-value \\
\hline Age [mean (1st, 3rd QU)] & $68.66(61,76)$ & $67.66(60,76)$ & 0.3496 \\
\hline Tumor stage (I/II/III/IV) & $0 / 41 / 67 / 48$ & $2 / 89 / 71 / 85$ & 0.3444 \\
\hline Grade (T1/T2/T3/T4) & $1 / 35 / 90 / 19$ & $3 / 84 / 104 / 36$ & 0.1084 \\
\hline Metastasis (Negative/Positive) & $60 / 5$ & $135 / 6$ & 0.3606 \\
\hline Lymph node (Positive/Negative) & $46 / 96$ & $82 / 139$ & 0.1604 \\
\hline Neoplasm (High/Low) & $156 / 0$ & $225 / 21$ & $2.999 e-06$ \\
\hline P-value < 0.05 as threshold. & & & \\
\hline
\end{tabular}

\section{Confirmation Of Glucose Metabolism-related Genes Associated With Survival}

In the training set, by comparing patients with survival time less than 1 year and patients who lived longer than 3 years, 70 DEGs were screened out with $p<0.05$ as the threshold (see Additional file 1). Then, 101 genes significantly associated to patients' survival status were obtained from 606 glucose metabolismrelated genes by using univariate COX regression analysis in the second step (see Additional file 2). The 46 overlapping genes from the two steps above were survival-related DEGs. In the third step, by analyzing promoter methylation levels of the 46 survival-related DEGs, we detected 18 genes of which the expression levels were significantly correlated to corresponding promotor methylation strengths, regarding the numbers of significant probes/the numbers of non-significant probes $>2 / 3$ as the threshold (see Additional file 3). Thus, via the three steps above, a total of 18 target genes (FBP1, HIP1R, USF1, SLC45A3, SARS, LRRC59, TRAF5, PICK1, UCHL1, NUP188, COPS5, MGST2, SSTR5, ZAP70, SLC44A4, PYCR1, TXNIP and ELF3) associated with both patients' prognosis and DNA methylation status were selected as predictors for following further study (Fig. 3a). The regression coefficients ( $\beta$ ) of the 18 genes were obtained from the univariate Cox proportional hazard regression analysis (Fig. 3b). Genes with regression coefficient values higher than 1 were identified as risky factors while those with coefficient values lower than 1 were protective factors.

\section{Identification of an 18 gene-related risk signature for the prognosis of BLCA}

To predict the overall survival of BLCA patients, we built a risk score formula on the basis of the expressions and the regression coefficients of the 18 selected genes as follows: risk score $=$ 
$(0.852801514$ * expression level of FBP1 $)+(0.658588685$ * expression level of HIP1R $)+(0.632868909$ * expression level of USF1) $+(0.759835042$ * expression level of SLC45A3) + (1.746061357 * expression level of SARS $)+(1.53204174$ * expression level of LRRC59) + (0.636153332 * expression level of TRAF5) $+(0.662610091$ * expression level of PICK1 $)+(1.121981252$ * expression level of UCHL1) + $(1.421043199 *$ expression level of NUP188 $)+(0.584186664 *$ expression level of COPS5) + $(0.787686002$ * expression level of MGST2 $)+(0.527554635$ * expression of SSTR 5$)+(0.777212294$ * expression level of ZAP70 $)+(0.918628408$ * expression level of SLC44A4 $)+(1.153860484$ * expression level of PYCR1 $)+(0.880775148$ * expression level of TXNIP $)+(0.917167392$ * expression level of ELF3). We called the signature we established above the18-mRNA risk score.

After calculating the risk score of each patient according to their 18-mRNA expression signature in the training set, we divided patients into a high-risk group $(n=150)$ and a low-risk group $(n=150)$ with the median risk score as the cutoff(Fig. 4a). Each patient's survival time and ending point are shown in (Fig. 4b), which reveals remarkable worse prognosis in the high-risk group than in the low-risk group. The 18 selected genes' expression levels of each patient are shown in (Fig. 4c) and we found that risky and protective genes showed opposite expression patterns according to the 18-mRNA risk score. Higher levels of 5 genes (UCHL1, PYCR1, SARS, NUP188, LRRC59) were more likely to be expressed in the high-risk group than in the low-risk group while the other genes (FBP1, HIP1R,USF1, SLC45A3, TRAF5, PICK1, COPS5, MGST2, SSTR5, ZAP70, SLC44A4, TXNIP, ELF3) were in the opposite situation, which was similar to our previous result (Fig. 3b).

We then carried out Kaplan-Meier analysis of overall survival for patients in the training set, which suggested great performance of the risk model in dividing patients into high-risk and low-risk $(p=0.0015)$ (Fig. 4d). In order to confirm the predictive ability of this risk formula, we calculated the 18-mRNA risk score of each patient in the validation set and stratified the patients into the same subgroups by using the median risk score as the cutoff. Similar to the result in the training set, the overall survival rate of the high-risk group was significantly lower than that of the low-risk group in the validation set $(p=0.00024)$ (Fig. 4e).

ROC analyses for 1-year, 3-year and 5-year survival were respectively carried out in the training set and the validation set, respectively. The results were shown in Fig. 5a-f, which exhibited satisfying survival predictive ability of the 18-mRNA risk score signature. Moreover, the sensitivity and specificity were even higher for the validation set than for the training set, which provides us with more confidence in the prognostic signature we built.

\section{The 18-mRNA risk score signature was an independent prognostic factor for bladder cancer patients}

Multivariate Cox regression analysis was performed in the validation set to determine whether the prognostic signature we had previously built could function as an independent prognostic indicator. 
When we included age, neoplasm grade, pathological T grade, pathological $\mathrm{N}$ grade, pathological $\mathrm{M}$ grade and diagnostic tumor stage together with the 18-mRNA risk score into the Multivariate Cox regression model, the age $(P<0.0001, H R=1.033)$, pathological $N$ stage $(P=0.022, H R=1.195)$ and the 18-mRNA risk score signature $(P=0.004, H R=1.167)$ showed significance while the other clinicopathological factors showed no statistical significance (Table. 2). Thus, it was indicated that the signature we established might be an independent prognostic factor for BLCA patients.

Table 2

Multivariate Cox regression analysis of the18-mRNA signature and clinical characteristics predictive of overall survival.

\begin{tabular}{|lll|}
\hline Variable & HR & P-value \\
\hline Age & 1.033 & $<0.001$ \\
\hline Neoplasm grade & 0.668 & 0.594 \\
\hline Pathological M stage & 1.182 & 0.082 \\
\hline Pathological N stage & 1.195 & 0.022 \\
\hline Pathological T stage & 1.329 & 0.091 \\
\hline Tumor grade & 1.328 & 0.070 \\
\hline 18-mRNA risk score signature & 1.167 & 0.004 \\
\hline HR: hazard ratio; P-value $<0.05$ as the criteria. \\
\hline
\end{tabular}

\section{GSEA analysis between high-risk patients and low-risk patients}

GSEA analysis result showed pathways and functions including wnt/beta-catenin-independent signaling pathway were significantly enriched in the high-risk group (Fig. 6a-c), which might provide us with insights into cancer progression and potential molecular mechanisms.

\section{Weighted Gene Co-expression Network Analysis (wgcna)}

With 24286 genes were adopted in WGCNA network construction, including mRNAs, miRNAs and IncRNAs, 34 modules in total were obtained. Among the 34 modules, 9 genes from the 18 glucose metabolism-related genes were identified as hub genes which were linked to 3 modules of co-expressive genes (Fig. 6d).

\section{Functional characteristics of UCHL1 and PYCR1}


In order to further validate our bioinformatic analyses, we tended to select two risky genes from the 18 genes to conduct cell functional experiments by using siRNAs to silence their expressions. Via the process of analyzing DNA methylation status of survival-related DEGs, we found the promotor methylation strengths of UCHL1 and PYCR1 were extraordinarily related to their own expressions. At the same time, no studies have so far reported the functions that these two genes play on bladder cancer cells. With great interest, we performed functional validations to determine the impacts that the two genes might have on bladder cancer cell lines, UM-UC-3 and T24. qRT-PCR results confirmed the knockdowns of UCHL1 and PYCR1 by siRNAs at mRNA level, respectively (Fig. 7A-B). Then, the proliferation ability, migration and invasiveness of BLCA cells were proved to be obviously inhibited after downregulating the expression of UCHL1 or of PYCR1 (Fig. 7C-D). Via performing western blotting, we noticed that silencing UCHL1 or PYVR1 resulted in downregulation of the expressions $\mathrm{N}$-cadherin and CyclinD1 at protein level, which suggested that the two genes might promote epithelial-mesenchymal transition (EMT)of bladder cancer cells and accelerate proliferation by regulating cell cycle (Fig. 7E). Meanwhile, no significant changes were detected in beta-catenin protein level, which was accordant to the GSEA result (Fig. 7E). In general, the results above provided us with not only experimental basis to partially validate our bioinformatic analyses but also with further insights to molecular functions and mechanisms for further research.

\section{Discussion}

Recently, several studies have applied gene expression signature to predict characteristics or prognosis for urinary bladder cancer. For example, a 20-gene signature strongly linked to bladder cancer development was established [28] and a 5-gene signature was developed for prediction of progression of T1G3 bladder cancer [29]; researchers previously reported a four-gene signature to directly predict OS of urinary bladder cancer patients [30]. However, a majority of these genes belong to various categories and owns different properties. On the other hand, it's necessary and urgent to explore new schemes for cancer prognosis with an easier access to gene abnormality detection in individuals, which will enhance the velocity and efficiency of translation from basic research to clinical application. A large number of studies have provided deep insights into molecular mechanisms of glucose metabolism alterations in cancers [8-12], by which we are more convinced of the critical role glucose metabolism plays in malignant tumors. Meanwhile, with abnormal alterations widely appearing in different cancers, DNA methylation has been recently analyzed by computational models to discover potential biomarkers for prediction of early diagnosis or prognosis of cancers [18-20]. Here, we selected genes significantly correlated to their promotor methylation status as well as to the OS of bladder cancer after conducting full-scale analyses of glucose metabolism-related genes.

In our study, from 606 glucose metabolism-related genes in 12 pathways, we first screened out 18 target genes, of which expressions were significantly correlated both to promotor methylation strengths and bladder patients' survival. Then, a signature based on the expressions of these 18 genes were constructed, which showed great survival predictive ability both in training and validation sets and was believed to act as a potential independent indicator for prognosis of bladder patients. 
Among the 18 genes, FBP1 has been reported to serve as a potential biomarker for molecular subtypes of bladder cancer for its participation in glycolysis flux and gluconeogenesis [31]; MGST2 could be induced in bladder cancer cells by Bacillus Calmette-Guérin (BCG) internalization and thus it might act as a novel biomarker of the response to BCG immunotherapy for bladder cancer [32]; TXNIP negatively regulates bladder carcinogenesis by attenuating SDF-1-CXCR4-induced ERK activation [33]; ELF3 can protects bladder cancer cells from epithelial-mesenchymal transition [34]. These above are highly in accordance to our results, supporting the validity of our study strategy. As for the other genes, ubiquitin carboxylterminal esterase L1 (UCHL1) and pyrroline-5-carboxylate reductase 1 (PYCR1) aroused our interest with their expressions extremely correlated to their promotor methylation strengths. Besides, functions of the two genes so far have not been systemically reported in urinary bladder cancer. Relevant functional experiments in bladder cancer cell lines revealed the roles UCHL1 and PYCR1 played in promoting cells' proliferation, migration and invasiveness by possibly regulating cell cycle and EMT, which was basic accordant with our bioinformatic analyses. The role of PYCR1 as an oncogene has been demonstrated in several other types of cancers via different molecular mechanisms including regulating P38 MAPK and NF-kB signaling pathway [35-38]. Besides, PYCR1 can be regulated by microRNAs in some cancers [3738]. What appeals to us is that despite participation in glucose metabolism, PYCR1 also plays a role in proline metabolism [39] and thus it's worth more comprehensive insights into its molecular functions. Different from PYCR1, whether UCHL1 is an oncogene or a tumor-suppressor depends on types of cancer [40-44]. However, similar to our findings, an article indicated that prognosis of renal cell carcinoma was associated with alterations of UCHL1 promoter methylation [44]. Moreover, according to the GSEA results, the high-risk patients significantly enriched genes involved in wnt/beta-catenin-independent signaling pathway, PCP pathway and protein folding, which were demonstrated to be linked to a variety of cancers [45-50]. Via experiments, we proved that downregulation of UCHL1 or PYCR1 indeed resulted in no obvious changes in beta-catenin protein level. Three modules of co-expression genes aimed at 9 hub genes were identified by WGCNA and along with GSEA results and it's worth further and deeper explorations for molecular mechanism demonstration and clinical application. Generally speaking, with functional experiment validations of the two genes, we have more confidence not only in UCHL1 and PYCR1 as potential biomarkers but also in the signature we constructed as a prognosis predictor for bladder cancer patients.

In our study, the 18 survival-related genes were all characterized by glucose metabolism participation and were promotor methylation associated, which hasn't been reported before. Currently, most cases of bladder cancer are diagnosed at advanced stages so that patients fail to receive curative surgery. Chemotherapy has been the main option for bladder cancer treatment [51], but the impact it has on overall survival is still limited [2-4]. Our study provides urologists and researchers with a potential new method for bladder cancer prognosis evaluation by DNA methylation monitoring with its advantage of stable existence in plasma; on the other hand, a rationale for treating bladder cancer patients by targeting metabolism is worth further consideration. However, a few limitations of our study should be emphasized. First, our bioinformatic analyses were limited within TCGA database and we only conducted functional experiments aimed at two risky genes that were outstandingly methylation-associated. 
Second, despite retrospective validations, our results need to be validated in further prospective investigations.

In summary, we developed a glucose metabolism gene-related signature that could potentially act as an independent prognosis predictor for bladder cancer patients. Further explorations into molecular mechanisms will reveal how these genes regulate glucose metabolism in cancer progression and how the expressions of the genes are modulated by promotor methylations, which can provide prospective and inspiration for future strategies for bladder cancer diagnosis, progression monitoring and even treatments.

\section{Conclusions}

In this study, we were convinced that glucose metabolism played a critical role in urinary bladder cancer. 18 glucose metabolism-related genes were filtered, which were significantly linked to bladder cancer patients' survival status and regulated by DNA methylation. A prognostic signature based on the expressions of the 18 genes was identified as a potential independent factor for predicting the prognosis of bladder cancer patients. Two risky genes from the 18 genes, UCHL1 and PYCR1, were found to be able to promote bladder cancer cells' proliferation, migration and evasion via cell functional experiments, which could further verify our bioinformatic analyses. In general, the 18 gene-related prognostic signature has a potential to expand the vision of prognosis prediction and even treatment for urinary bladder cancer patients.

\section{Abbreviations}

AUC: area under the curve; BLCA: Bladder Urothelial Carcinoma; ES: enrichment score; FDR: false discovery rate; FWER: family-wise error rate; GSEA: gene set enrichment analysis; HR: hazard ratios; OS: overall survival; ROC: receiver operating characteristic; TCGA: The Cancer Genome Atlas; WGCNA: weighted gene co-expression network analysis.

\section{Declarations}

\section{Acknowledgements}

Not applicable.

\section{Authors' contributions}

ZL, TS and ZZ conceived the idea for the paper and performed the bioinformatic analyses. ZL performed the cell functional validations and was a major contributor in writing the manuscript. CK provided fund support. CK was involved in drafting the manuscript or revising it critically for important intellectual content. All authors read and approved the final manuscript. 


\section{Funding}

This work was supported by Shenyang Plan Project of Science and Technology (Grant No. F19-112-4098), National key R \& D plan key research projects of precision medicine (2017YFC0908000), China Medical University's 2019 discipline promotion program. The authors declare no competing financial interests. Funding agency did not participate in the design of the study and collection, analysis and interpretation of data and in writing the manuscript.

\section{Availability of data and materials}

Not applicable.

\section{Ethics approval and consent to participate}

Not applicable.

\section{Consent for publication}

Not applicable.

\section{Competing interests}

The authors declare that they have no competing interests.

\section{Author details}

${ }^{1}$ Department of Urology, Fist Hospital of China Medical University; School of China Medical University; No. 155 Nanjing North Street, Heping District, Shenyang City, Liaoning Province, 110004, P.R. China.

2 Department of Obstetrics and Gynecology, Shengjing Hospital of China Medical University, Shenyang, Liaoning 110004, P.R. China.

\section{References}

1. Siegel RL, Miller KD, Jemal A: Cancer statistics, 2018. CA Cancer J Clin 2018, 68:7-30.

2. Ribas A, Tumeh PC: The future of cancer therapy: selecting patients likely to respond to PD1/L1 blockade. Clin Cancer Res 2014, 20:4982-4984.

3. Oing C, Rink M, Oechsle K, Seidel C, von Amsberg G, Bokemeyer C: Second Line Chemotherapy for Advanced and Metastatic Urothelial Carcinoma: Vinflunine and Beyond-A Comprehensive Review of the Current Literature. J Urol 2016, 195:254-263.

4. van Kessel KE, Zuiverloon TC, Alberts AR, Boormans JL, Zwarthoff EC: Targeted therapies in bladder cancer: an overview of in vivo research. Nat Rev Urol 2015, 12:681-694. 
5. Vander Heiden MG, Cantley LC, Thompson CB: Understanding the Warburg effect: the metabolic requirements of cell proliferation. Science 2009, 324:1029-1033.

6. Cantor JR, Sabatini DM: Cancer cell metabolism: one hallmark, many faces. Cancer Discov 2012, 2:881-898.

7. Warburg O: On the origin of cancer cells. Science 1956, 123:309-314.

8. Stine ZE, Walton ZE, Altman BJ, Hsieh AL, Dang CV: MYC, Metabolism, and Cancer. Cancer Discov 2015, 5:1024-1039.

9. Xiao ZD, Han L, Lee H, Zhuang L, Zhang Y, Baddour J, Nagrath D, Wood CG, Gu J, Wu X, et al: Energy stress-induced IncRNA FILNC1 represses c-Myc-mediated energy metabolism and inhibits renal tumor development. Nat Commun 2017, 8:783.

10. Velpula KK, Bhasin A, Asuthkar S, Tsung AJ: Combined targeting of PDK1 and EGFR triggers regression of glioblastoma by reversing the Warburg effect. Cancer Res 2013, 73:7277-7289.

11. Zhang Y, Yang JM: Altered energy metabolism in cancer: a unique opportunity for therapeutic intervention. Cancer Biol Ther 2013, 14:81-89.

12. Yang W, Zheng Y, Xia Y, Ji H, Chen X, Guo F, Lyssiotis CA, Aldape K, Cantley LC, Lu Z: ERK1/2dependent phosphorylation and nuclear translocation of PKM2 promotes the Warburg effect. Nat Cell Biol 2012, 14:1295-1304.

13. Ehrlich M: DNA methylation in cancer: too much, but also too little. Oncogene 2002, 21:5400-5413.

14. Eden A, Gaudet F, Waghmare A, Jaenisch R: Chromosomal instability and tumors promoted by DNA hypomethylation. Science 2003, 300:455.

15. Moore LD, Le T, Fan G: DNA methylation and its basic function. Neuropsychopharmacology 2013, 38:23-38.

16. Yang B, Guo M, Herman JG, Clark DP: Aberrant promoter methylation profiles of tumor suppressor genes in hepatocellular carcinoma. Am J Pathol 2003, 163:1101-1107.

17. Si JG, Su YY, Han YH, Chen RH: Role of RASSF1A promoter methylation in the pathogenesis of ovarian cancer: a meta-analysis. Genet Test Mol Biomarkers 2014, 18:394-402.

18. Pan Y, Liu G, Zhou F, Su B, Li Y: DNA methylation profiles in cancer diagnosis and therapeutics. Clin Exp Med 2018, 18:1-14.

19. Hao X, Luo H, Krawczyk M, Wei W, Wang W, Wang J, Flagg K, Hou J, Zhang H, Yi S, et al: DNA methylation markers for diagnosis and prognosis of common cancers. Proc Natl Acad Sci U S A 2017, 114:7414-7419.

20. Zhang $E$, Hou X, Hou B, Zhang M, Song Y: A risk prediction model of DNA methylation improves prognosis evaluation and indicates gene targets in prostate cancer. Epigenomics 2020, 12:333-352.

21. Laird PW: The power and the promise of DNA methylation markers. Nat Rev Cancer 2003, 3:253-266.

22. Zheng Y, Huang Q, Ding Z, Liu T, Xue C, Sang X, Gu J: Genome-wide DNA methylation analysis identifies candidate epigenetic markers and drivers of hepatocellular carcinoma. Brief Bioinform 2018, 19:101-108. 
23. Cheng J, Wei D, Ji Y, Chen L, Yang L, Li G, Wu L, Hou T, Xie L, Ding G, et al: Integrative analysis of DNA methylation and gene expression reveals hepatocellular carcinoma-specific diagnostic biomarkers. Genome Med 2018, 10:42.

24. Xu RH, Wei W, Krawczyk M, Wang W, Luo H, Flagg K, Yi S, Shi W, Quan Q, Li K, et al: Circulating tumour DNA methylation markers for diagnosis and prognosis of hepatocellular carcinoma. Nat Mater 2017, 16:1155-1161.

25. Song LL, Li YM: Current noninvasive tests for colorectal cancer screening: An overview of colorectal cancer screening tests. World J Gastrointest Oncol 2016, 8:793-800.

26. Langfelder $P$, Horvath $S$ : WGCNA: an R package for weighted correlation network analysis. BMC Bioinformatics 2008, 9:559.

27. Merico D, Isserlin R, Stueker O, Emili A, Bader GD: Enrichment map: a network-based method for geneset enrichment visualization and interpretation. PLoS One 2010, 5:e13984.

28. Smith SC, Baras AS, Dancik G, Ru Y, Ding KF, Moskaluk CA, Fradet Y, Lehmann J, Stockle M, Hartmann A, et al: A 20-gene model for molecular nodal staging of bladder cancer: development and prospective assessment. Lancet Oncol 2011, 12:137-143.

29. van der Heijden AG, Mengual L, Lozano JJ, Ingelmo-Torres M, Ribal MJ, Fernandez PL, Oosterwijk E, Schalken JA, Alcaraz A, Witjes JA: A five-gene expression signature to predict progression in T1G3 bladder cancer. Eur J Cancer 2016, 64:127-136.

30. Chen S, Zhang N, Shao J, Wang T, Wang X: A novel gene signature combination improves the prediction of overall survival in urinary bladder cancer. J Cancer 2019, 10:5744-5753.

31. Stachowiak M, Szymanski M, Ornoch A, Jancewicz I, Rusetska N, Chrzan A, Demkow T, Siedlecki JA, Sarnowski TJ, Sarnowska E: SWI/SNF chromatin remodeling complex and glucose metabolism are deregulated in advanced bladder cancer. IUBMB Life 2020.

32. Rahmat JN, Esuvaranathan K, Mahendran R: Bacillus Calmette-Guerin induces rapid gene expression changes in human bladder cancer cell lines that may modulate its survival. Oncol Lett 2018, 15:9231-9241.

33. Nishizawa K, Nishiyama H, Matsui Y, Kobayashi T, Saito R, Kotani H, Masutani H, Oishi S, Toda Y, Fujii N, et al: Thioredoxin-interacting protein suppresses bladder carcinogenesis. Carcinogenesis 2011, 32:1459-1466.

34. Gondkar K, Patel K, Krishnappa S, Patil A, Nair B, Sundaram GM, Zea TT, Kumar P: E74 like ETS transcription factor 3 (ELF3) is a negative regulator of epithelial- mesenchymal transition in bladder carcinoma. Cancer Biomark 2019, 25:223-232.

35. Yan K, Xu X, Wu T, Li J, Cao G, Li Y, Ji Z: Knockdown of PYCR1 inhibits proliferation, drug resistance and EMT in colorectal cancer cells by regulating STAT3-Mediated p38 MAPK and NF-kappaB signalling pathway. Biochem Biophys Res Commun 2019, 520:486-491.

36. Zeng T, Zhu L, Liao M, Zhuo W, Yang S, Wu W, Wang D: Knockdown of PYCR1 inhibits cell proliferation and colony formation via cell cycle arrest and apoptosis in prostate cancer. Med Oncol 2017, 34:27. 
37. Sun T, Song Y, Yu H, Luo X: Identification of IncRNA TRPM2-AS/miR-140-3p/PYCR1 axis's proliferates and anti-apoptotic effect on breast cancer using co-expression network analysis. Cancer Biol Ther 2019, 20:760-773.

38. Wang D, Wang L, Zhang Y, Yan Z, Liu L, Chen G: PYCR1 promotes the progression of non-small-cell lung cancer under the negative regulation of miR-488. Biomed Pharmacother 2019, 111:588-595.

39. Chen S, Yang X, Yu M, Wang Z, Liu B, Liu M, Liu L, Ren M, Qi H, Zou J, et al: SIRT3 regulates cancer cell proliferation through deacetylation of PYCR1 in proline metabolism. Neoplasia 2019, 21:665675.

40. Moore MD, Finnerty B, Gray KD, Hoda R, Liu YF, Soong L, Beninato T, Rao R, Zarnegar R, Fahey TJ, 3rd: Decreased UCHL1 expression as a cytologic biomarker for aggressive behavior in pancreatic neuroendocrine tumors. Surgery 2018, 163:226-231.

41. Kwan SY, Au-Yeung CL, Yeung TL, Rynne-Vidal A, Wong KK, Risinger JI, Lin HK, Schmandt RE, Yates MS, Mok SC, Lu KH: Ubiquitin Carboxyl-Terminal Hydrolase L1 (UCHL1) Promotes Uterine Serous Cancer Cell Proliferation and Cell Cycle Progression. Cancers (Basel) 2020, 12.

42. Nakao K, Hirakawa T, Suwa H, Kogure K, Ikeda S, Yamashita S, Minegishi T, Kishi H: High Expression of Ubiquitin C-terminal Hydrolase L1 Is Associated With Poor Prognosis in Endometrial Cancer Patients. Int J Gynecol Cancer 2018, 28:675-683.

43. Gu Y, Lv F, Xue M, Chen K, Cheng C, Ding X, Jin M, Xu G, Zhang Y, Wu Z, et al: The deubiquitinating enzyme UCHL1 is a favorable prognostic marker in neuroblastoma as it promotes neuronal differentiation. J Exp Clin Cancer Res 2018, 37:258.

44. Kagara I, Enokida H, Kawakami K, Matsuda R, Toki K, Nishimura H, Chiyomaru T, Tatarano S, Itesako T, Kawamoto K, et al: $\mathrm{CpG}$ hypermethylation of the UCHL1 gene promoter is associated with pathogenesis and poor prognosis in renal cell carcinoma. J Urol 2008, 180:343-351.

45. Flores-Hernandez E, Velazquez DM, Castaneda-Patlan MC, Fuentes-Garcia G, Fonseca-Camarillo G, Yamamoto-Furusho JK, Romero-Avila T, Garcia-Sainz JA, Robles-Flores M: Canonical and noncanonical Wnt signaling are simultaneously activated by Wnts in colon cancer cells. Cell Signal 2020:109636.

46. Chen J, Wang M, Xiang Y, Ru X, Ren Y, Liu X, Qiu L, Zhang Y: Nrf1 Is Endowed with a Dominant Tumor-Repressing Effect onto the Wnt/beta-Catenin-Dependent and Wnt/beta-Catenin-Independent Signaling Networks in the Human Liver Cancer. Oxid Med Cell Longev 2020, 2020:5138539.

47. Humphries AC, Mlodzik M: From instruction to output: Wnt/PCP signaling in development and cancer. Curr Opin Cell Biol 2018, 51:110-116.

48. Katoh M: WNT/PCP signaling pathway and human cancer (review). Oncol Rep 2005, 14:1583-1588.

49. Kaschula CH, Hunter R, Cotton J, Tuveri R, Ngarande E, Dzobo K, Schafer G, Siyo V, Lang D, Kusza DA, et al: The garlic compound ajoene targets protein folding in the endoplasmic reticulum of cancer cells. Mol Carcinog 2016, 55:1213-1228.

50. Van Drie JH: Protein folding, protein homeostasis, and cancer. Chin J Cancer 2011, 30:124-137. 
51. Felsenstein KM, Theodorescu D: Precision medicine for urothelial bladder cancer: update on tumour genomics and immunotherapy. Nat Rev Urol 2018, 15:92-111.

\section{Supplementary Information}

Additional file 1. xls. The result of DEGs identification. 70 DEGs were identified with $P<0.05$ as the criteria.

Additional file 2. xls. The positive result of the univariate Cox regression analysis. 101 genes were considered survival-related with $P<0.05$ as the criteria.

Additional file 3. xls. DNA methylation probes of the 18 genes. 18 DEGs' expressions were significantly related to their DNA methylation status considered the numbers of significant probes/the numbers of non-significant probes $>2 / 3$ as the threshold.

\section{Figures}

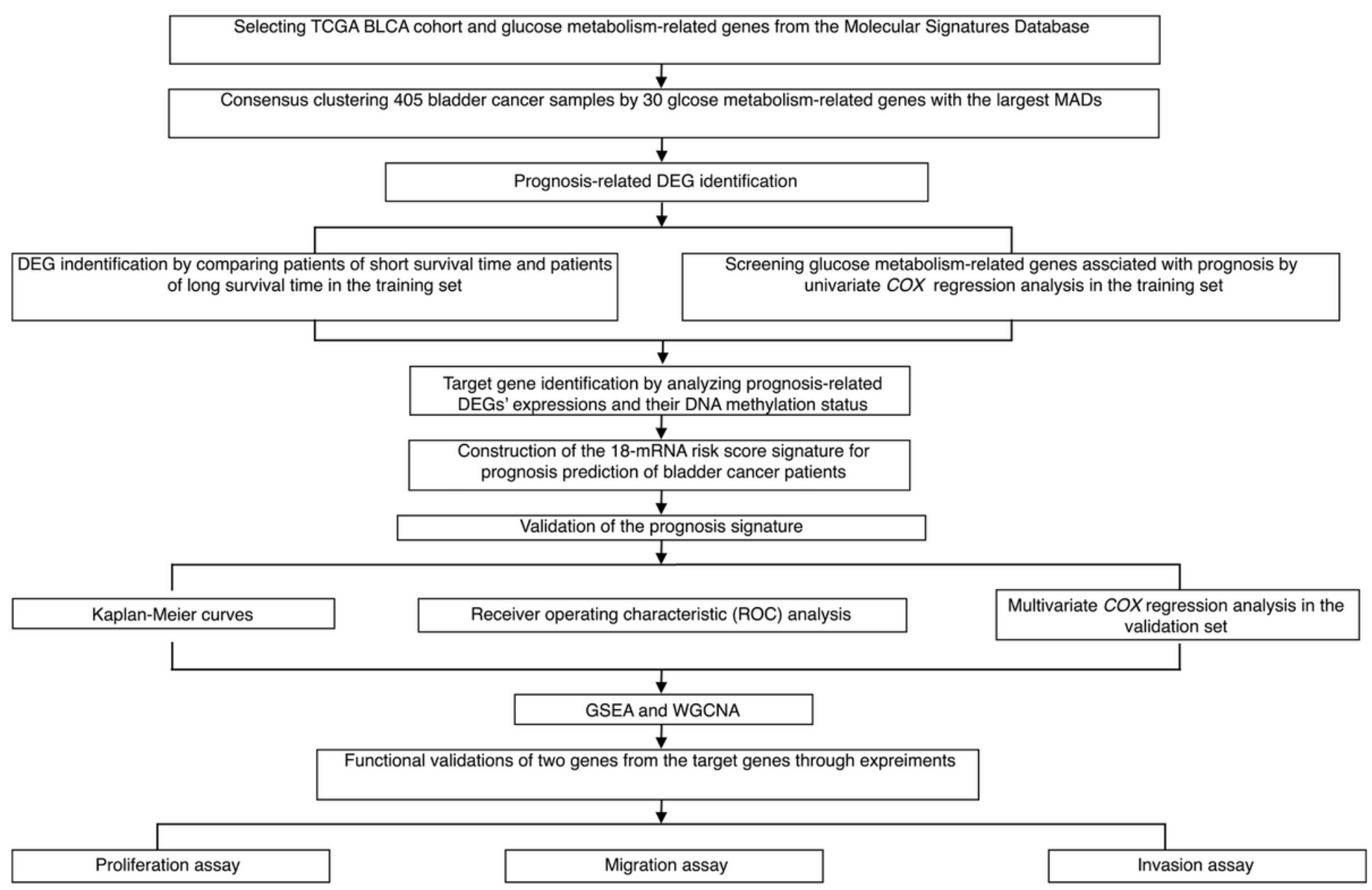

Figure 1

Pathway illustration of the present study. 
a

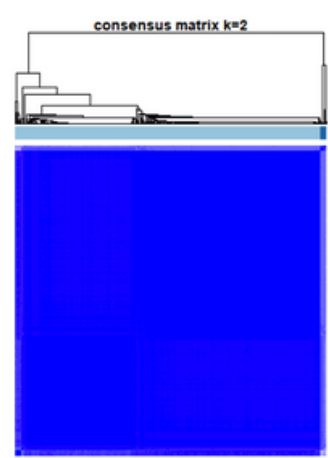

e
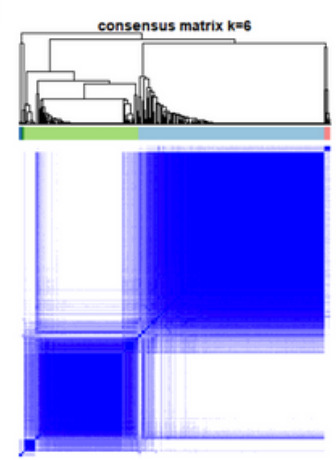

i

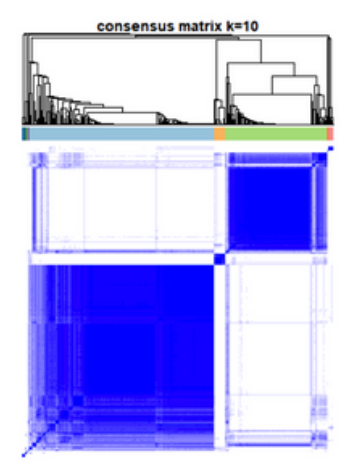

b

目

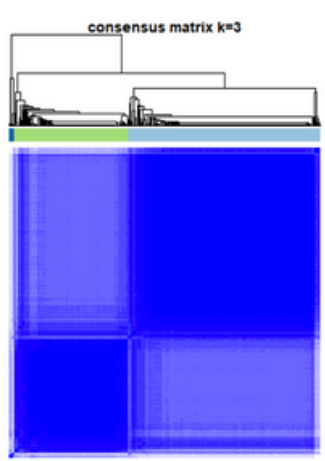

f
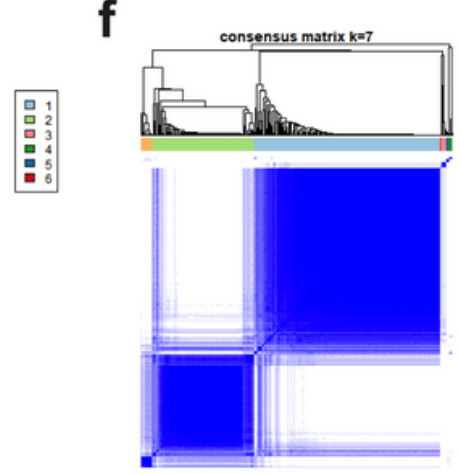

j
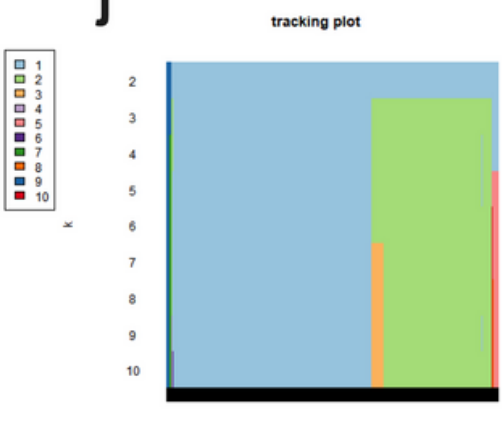

C
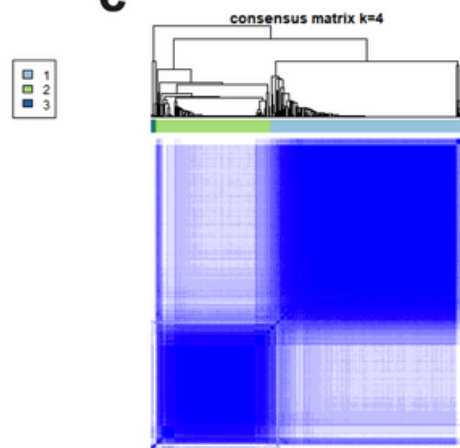

g
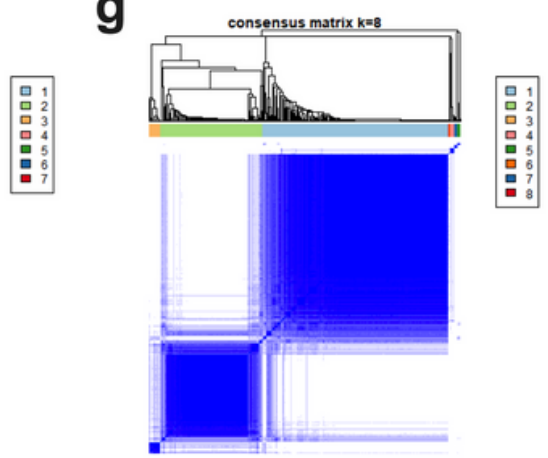

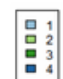

h

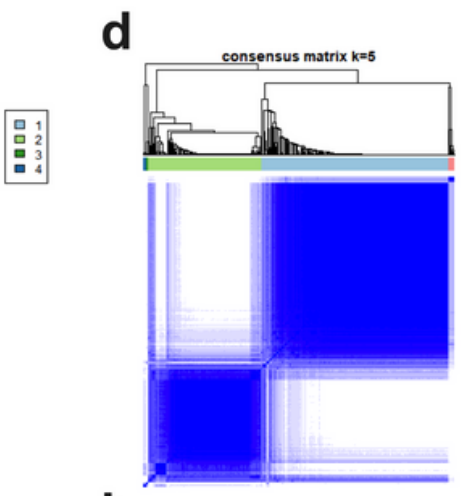

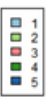

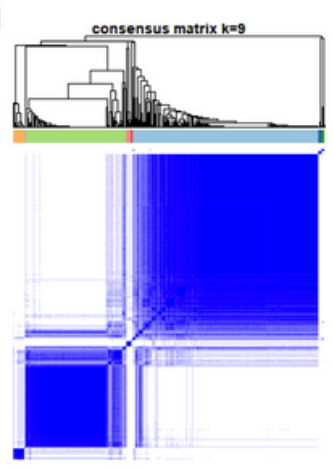

k

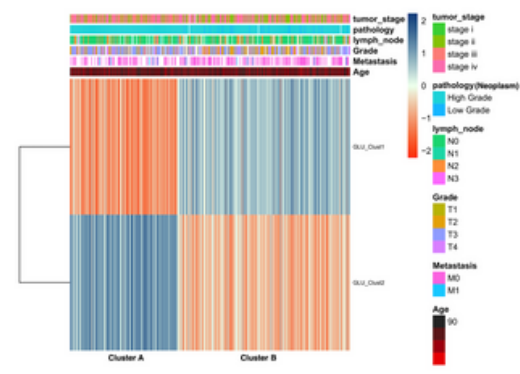

sameles

Figure 2

Classification of TCGA BLCA patients into clusters by 30 glucose metabolism-related genes of the largest MAD values. a-i The TCGA BLCA cohort was respectively divided into clusters with growing $k$ values by consensus clustering analysis. $\mathrm{j}$ The tracking plot for $\mathrm{k}=2$ to $10 . \mathrm{k}$ The TCGA BLCA cohort was divided into two clusters by two clusters of genes with clinicopathological characteristic comparisons. 
a

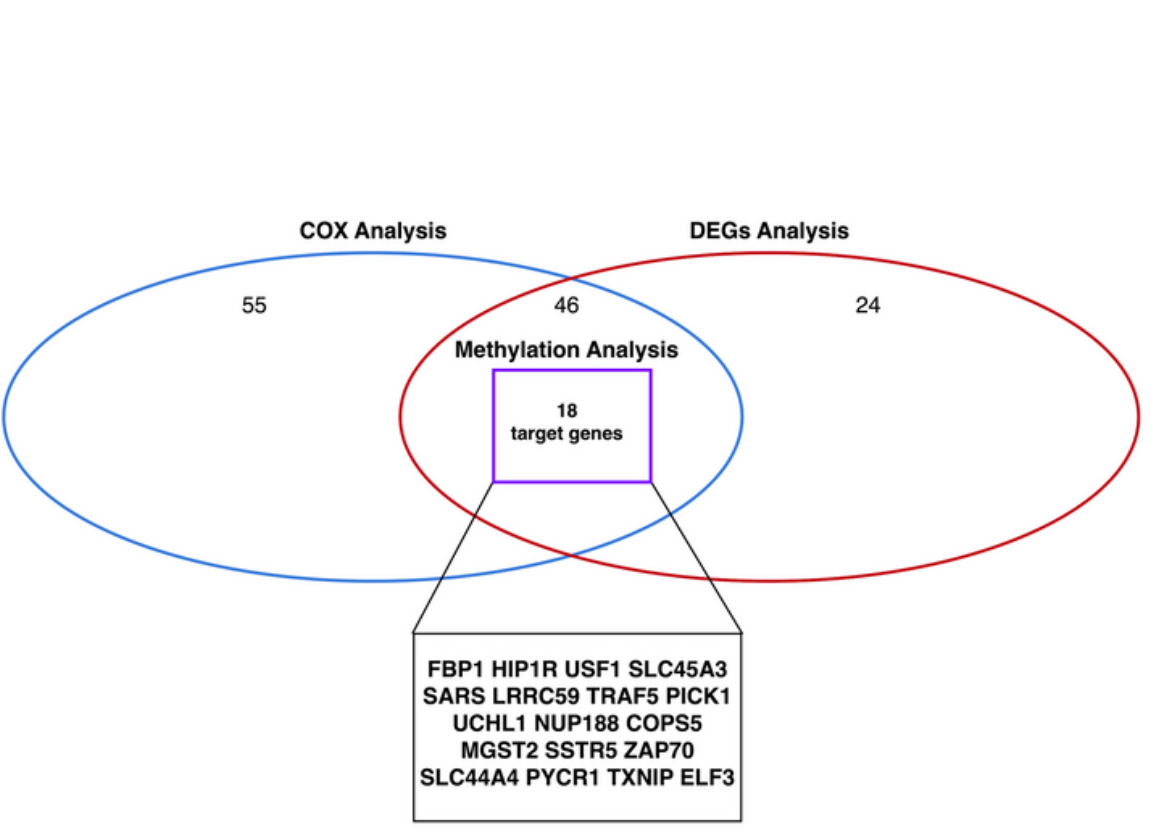

b

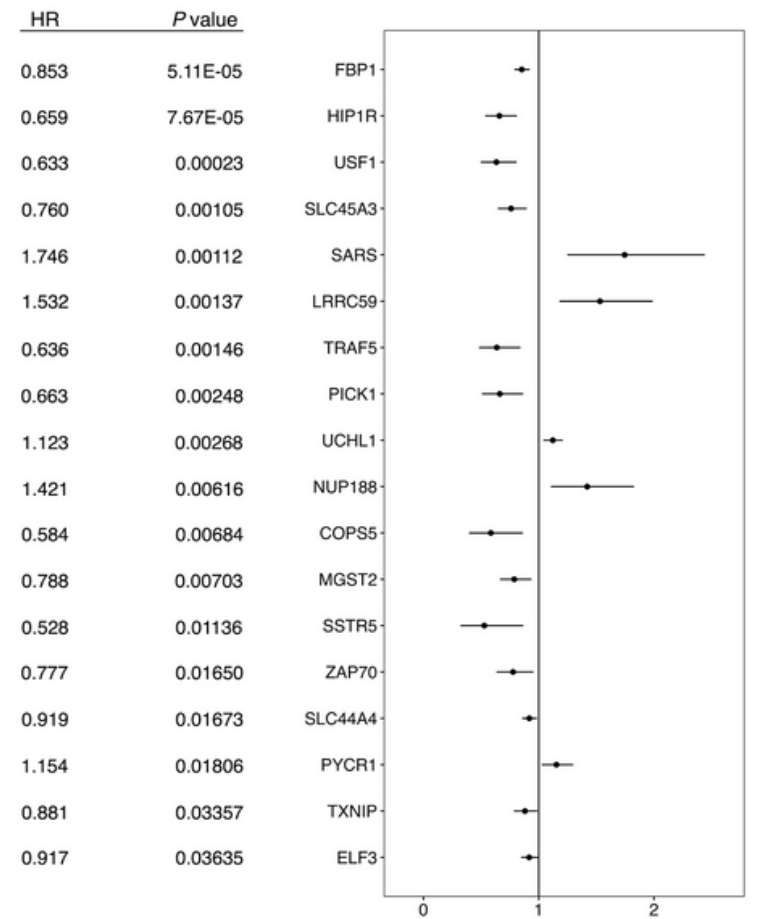

Figure 3

Identification of target genes. a Process of identifying survival-related genes with ex-pressions significantly correlated to corresponding promotor methylation 

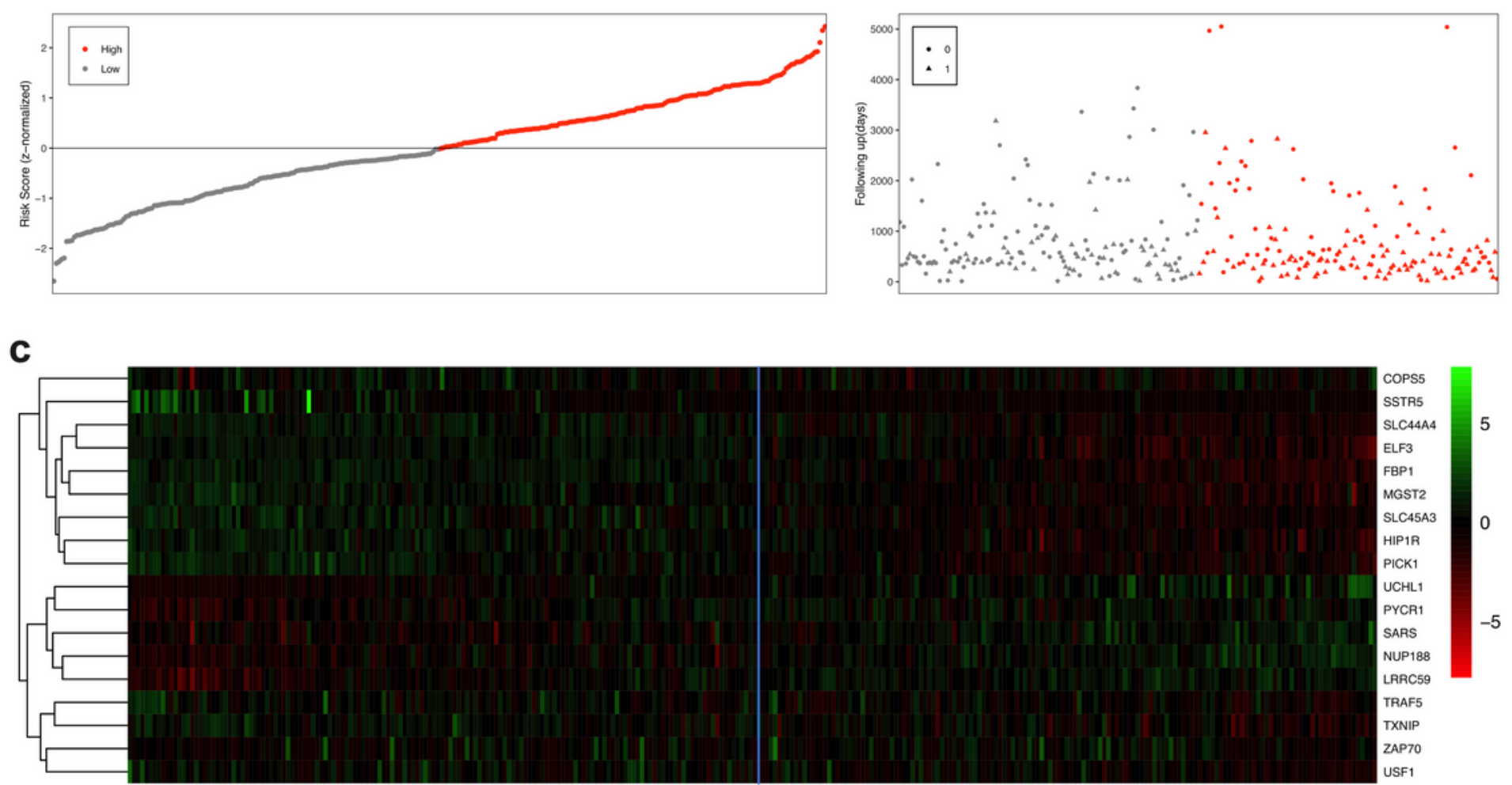

Patients with low risk values

Patients with high risk values

d

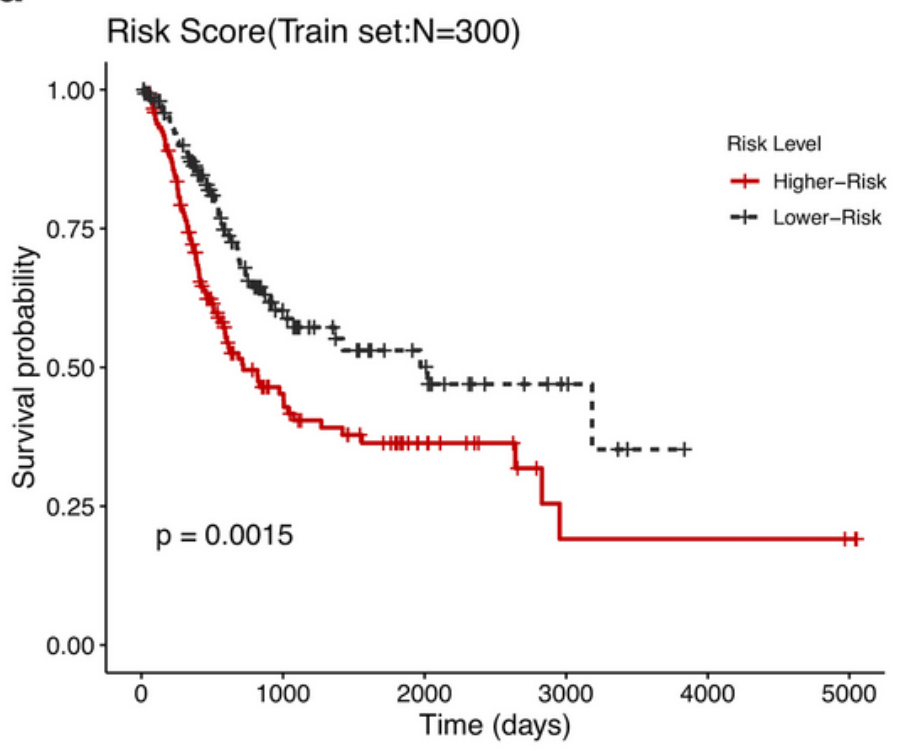

e

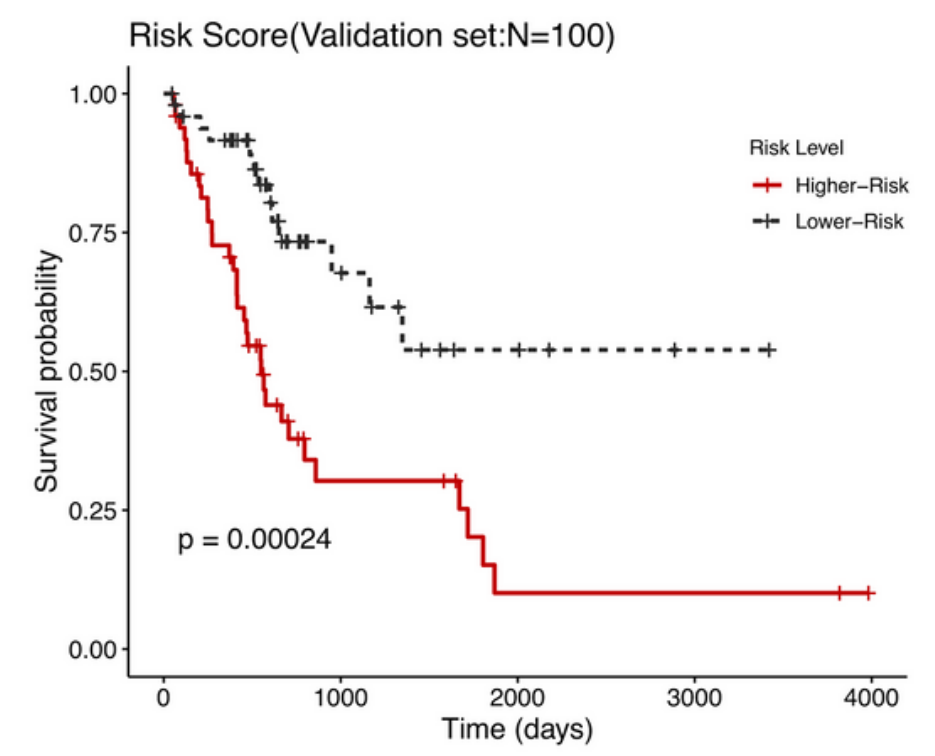

\section{Figure 4}

Construction of a prognostic signature for bladder cancer patients. a The distribution of risk scores. $b$ Patients' overall survival status distribution. A " 0 " represents survival at the patient's endpoint while a "1" represents a death. $c$ The heatmap of the expression levels of the 18 glucose metabolism-related genes. $d$ Kaplan-Meier analysis of OS for the training set $(n=300)$. e Kaplan-Meier analysis of OS for the validation set $(n=100)$. 
a

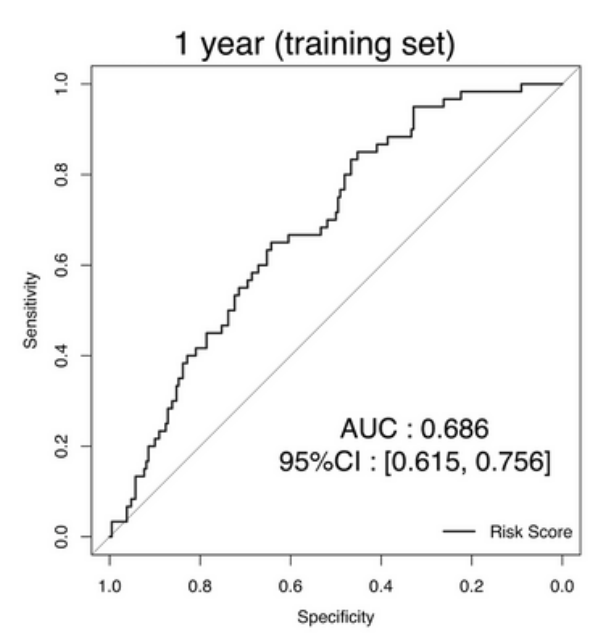

d

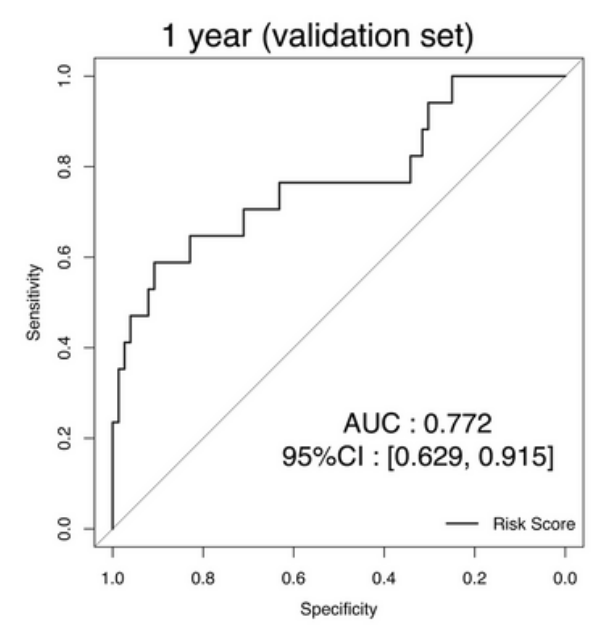

b

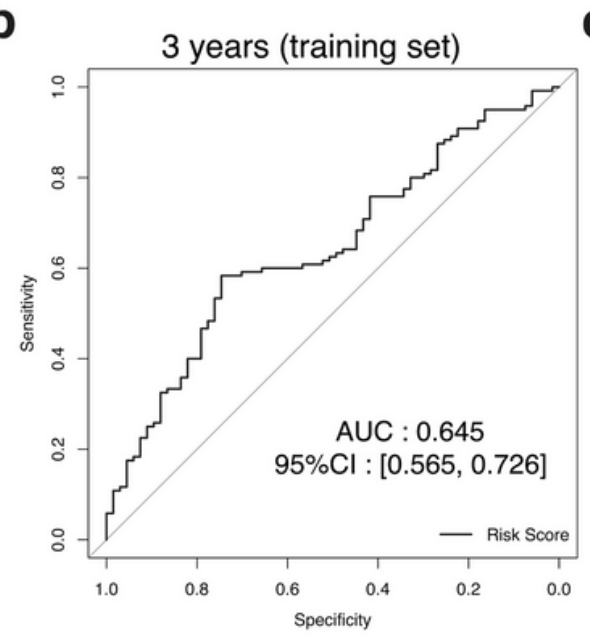

e

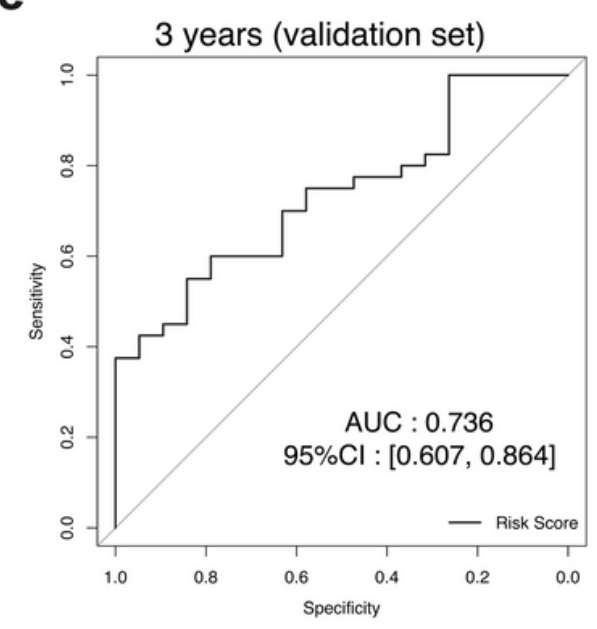

C

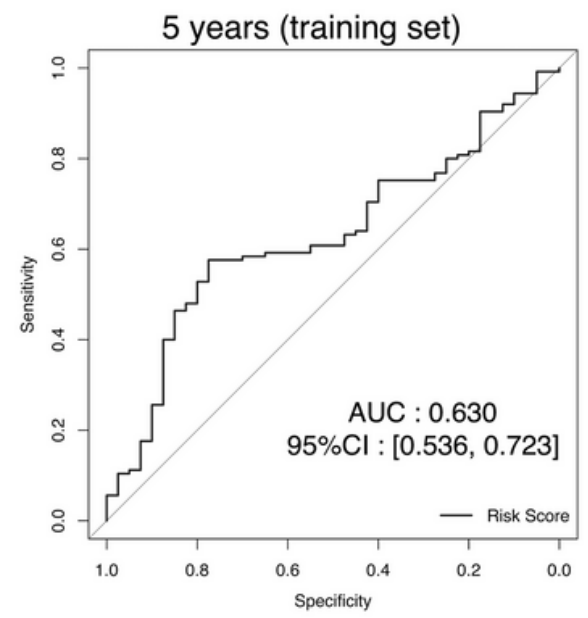

f

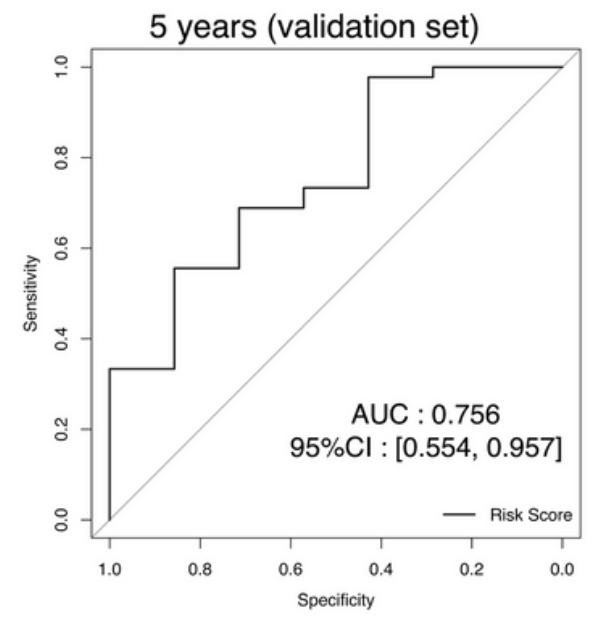

\section{Figure 5}

Identification of the sensitivity and the specificity of the 18-mRNA risk score signature. a-c ROC analyses at 1-year, 3-year and 5-year overall survival in the training set for the 18-mRNA signature. d-f ROC analyses at 1-year, 3-year and 5-year overall survival in the validation set for the 18-mRNA signature. 
a

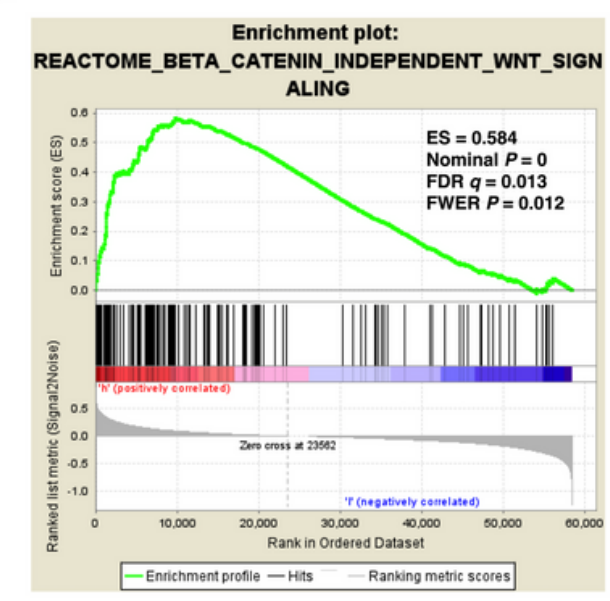

b

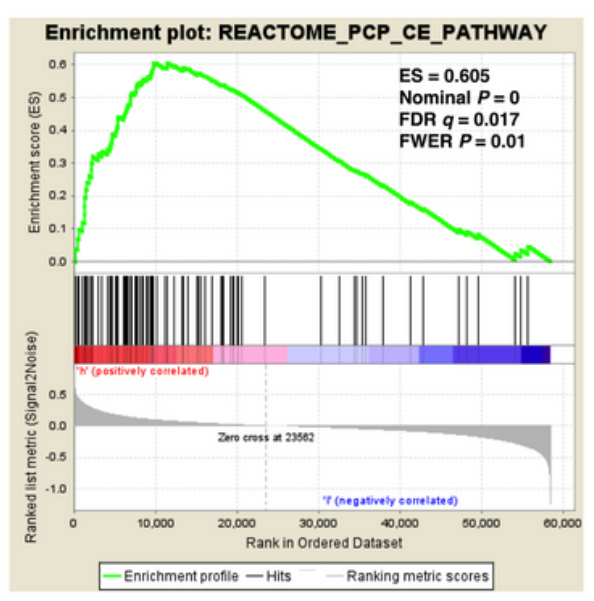

C

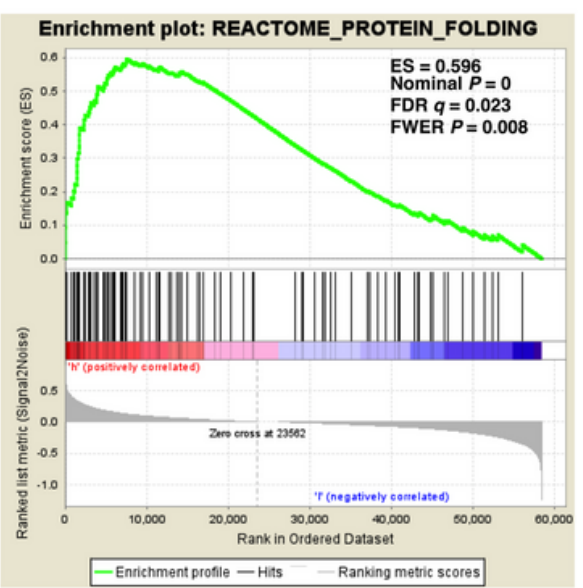

d
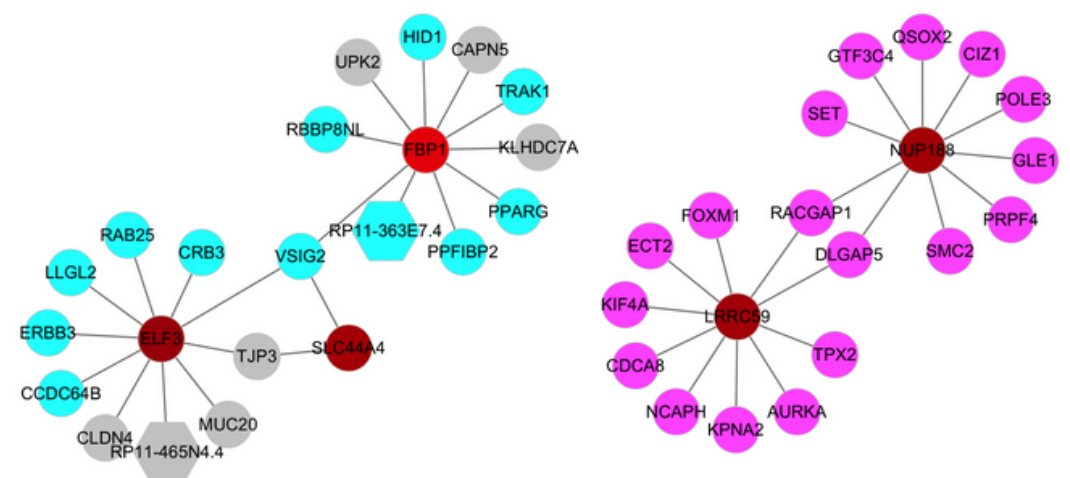

Anotation:

Red: Hub Gene

Others: module by WGCNA
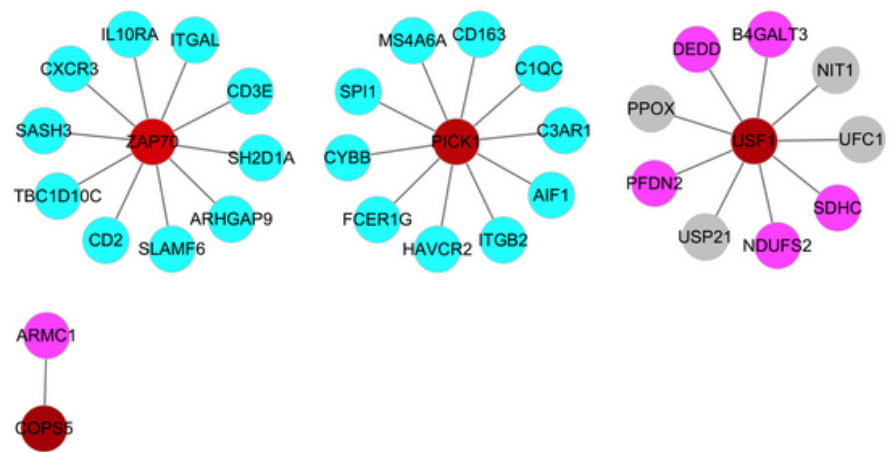

\section{Figure 6}

GSEA analysis and WGCNA analysis. a-c Identification of pathways and functions en-riched in the highrisk group by GSEA. d Hub gene identification and co-expression gene analysis by WGCNA. 
a

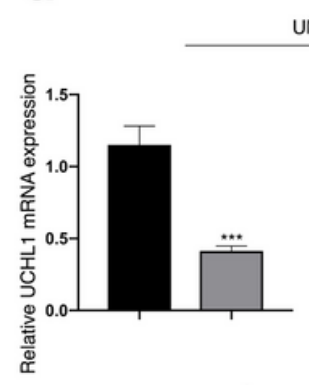

UM-UC-3
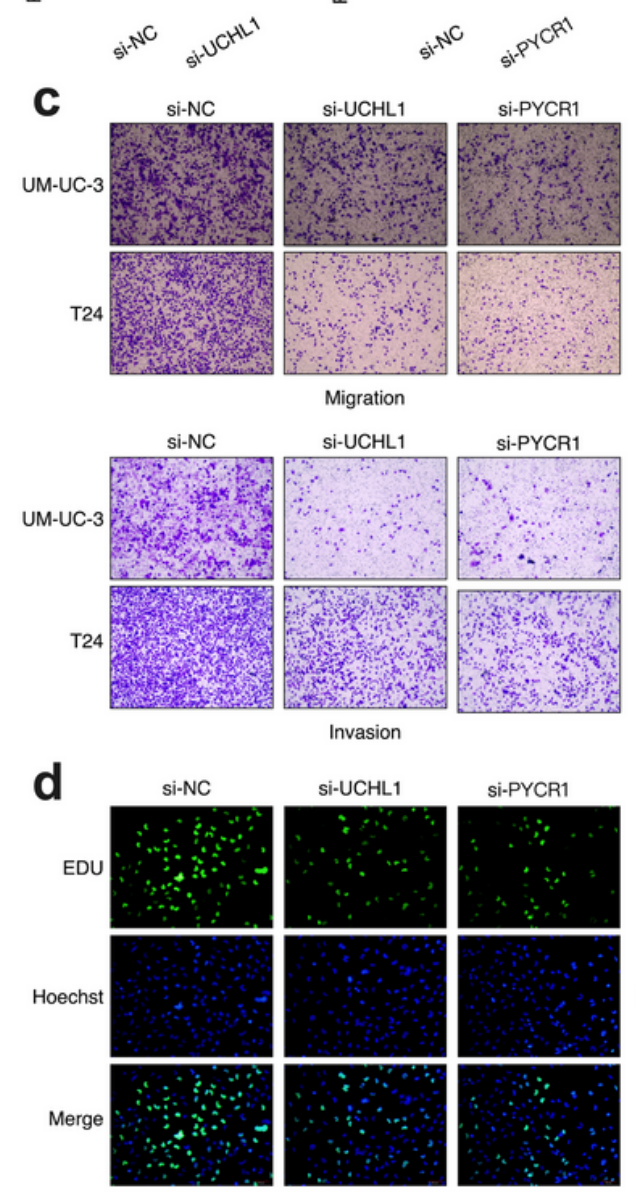

UM-UC-3 b
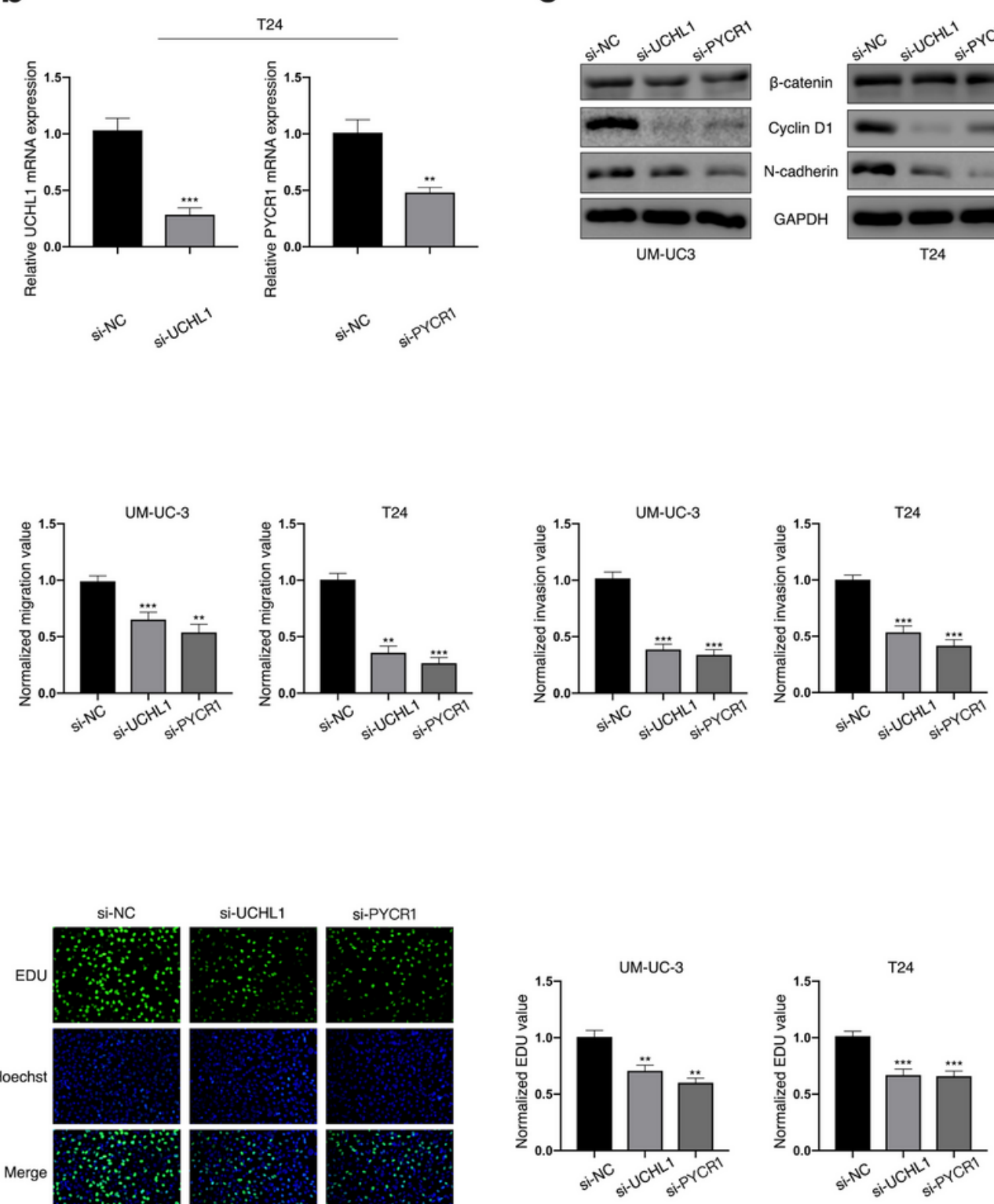

\section{Figure 7}

UCHL1 and PYCR1 respectively promotes bladder cancer cell migration, invasion and proliferation. a-b qRT-PCR results confirmed downregulations of UCHL1 and PYCR1 in UM-UC-3 and T24 by siRNAs. C Migration and invasion of UM-UC-3 and T24 after re-spectively silencing the expressions of UCHL1 and PYCR1. Data were analyzed by T-test and presented as the mean \pm standard deviation (SD). $d$ Proliferation of UM-UC-3 and T24 after respectively silencing the expressions of UCHL1 and PYCR1. Data were ana-lyzed by T-test and presented as the mean \pm standard deviation (SD). e Beta-catenin, cyclin D1 and $\mathrm{N}$-cadherin proteins of UM-UC-3 and T24 were detected after respectively silencing the expressions of UCHL1 and PYCR1. ${ }^{*}<<0.05 ; * \star P<0.01 ; * \star * P<0.001$.

\section{Supplementary Files}


This is a list of supplementary files associated with this preprint. Click to download.

- Additionalfile1.xlsx

- Additionalfile2.xlsx

- Additionalfile3.xlsx 